\title{
Some debugging strategies for assembler-based minicomputer operating systems
}

\author{
HOWARD L. KAPLAN \\ Computer Services, Addiction Research Foundation of Ontario, \\ 93 Russell Street, Toronto, Ontario, Canada M5S 2S1
}

\begin{abstract}
An assembler-based operating system can provide debugging features often found only on more advanced systems. These can include an interactive inspection program, an error-trap facility, and a real-time, hard copy trace of important program events. These features, which can reduce the number of recompilation cycles needed for debugging, have been implemented in the FRIVLOS operating system for a $32 \mathrm{~K}$ PDP-12.
\end{abstract}

One way to program a minicomputer for real-time control applications is to write in assembler. A different way is to write in a language such as real-time FOCAL or BASIC. There are many good reasons to continue to write in assembler: Assembler programs execute much faster, in general, than do programs written in high-level languages, and the structure of the particular paradigms to be implemented may not be easily expressible in the available languages. In particular, certain operations such as packing data or extracting single bits are handled more easily and directly with assembler rotate and mask instructions than with the arithmetic instructions of typical high-level languages. Nonetheless, writing in high-level languages has often been held as an ideal for experimental control. But when we consider some of the differences between FOCAL and assembler on a machine like a PDP-8 or PDP-12, we find that many of the differences are not inherent in the language structure but rather in the operating system support behind the languages. Assembler programming is traditionally an often-repeated cycle through editing, assembling, and debugging, each under the control of a separate program. On a paper-tape system this is now considered intolerable, and, while much faster on a diskor tape-based system with a display scope, such as a PDP-12, it is still a large number of different steps. In FOCAL, on the other hand, the editing, compilation, and debugging are all performed by a single program, greatly reducing both the time between recompilations and the fraction of the program that must be reprocessed, even if unchanged, to implement the next iteration.

In the autumn of 1973 , in B. B. Murdock's laboratory at the University of Toronto, we were faced with the development of a new software system for a 32K PDP12. This was an assembler-based operating system audaciously named FRIVLOS, a First-Rate Improved Verbal Learning Operating System. As in FOCAL, the part of the system seen by the typical user is only the tip of an

This research was supported by Grant 146 from the National Research Council of Canada. iceberg; the program written by the user works in conjunction with a much larger set of system-resident routines. These permanent routines are termed the operating system proper; the added material, the user program. Table 1 shows the allocation of system features into the eight $4 \mathrm{~K}$ memory fields.

In concept, FRIVLOS resembles languages such as PEPL (Getty, 1975). Program assembly is performed using the existing symbolic assembler DIAL-MS (which is similar to PAL III), but additional symbols are defined corresponding to resident system subroutines and parameters. Subroutine calls to the operating system are written as strings of such symbols, one per core location, and they can be freely intermixed with standard machine instructions. Table 2 shows that part of a typical user program which would display five words on the scope, each for $500 \mathrm{msec}$, with a $250-\mathrm{msec}$ ISI.

The first one and the last two instruction lines are straightforward PAL III code. The ACALL, MCALL, and WAIT instructions have been defined as subroutine calls. WGET, DISAPP, and BLANKR are the names (and hence addresses) of operating system routines. All other items are subroutine parameters, fetched and interpreted by the operating system, not executed directly. The system routines handle $\mathrm{I} / \mathrm{O}$, timing, randomization, subscripted array handling, and a number of special wordpool manipulation tasks. Not only do these routines reduce debugging time by freeing the user from the need to prepare his own routines for those tasks, they also in-

Table 1

Core Allocation of the FRIVLOS Operating System*

Field 0: $\quad 0000-5777$ Operating system routines 6000-7777 KNUCKLE

Field 1: $\quad 0000-0057,0160-0377$ Operating system routines

Fields 2, 3: $\quad$ User data

Field 4: $\quad 0000-3777$ Wordpool history counters 4000-5777 Character display co-ordinates tables 6000-7777 Tape transfer buffers

Fields 5, 6, 7: Wordpool storage (up to 2047 12-letter words)

* All addresses in octal notation. 
Table 2

Part of a Typical FRIVLOS User Program

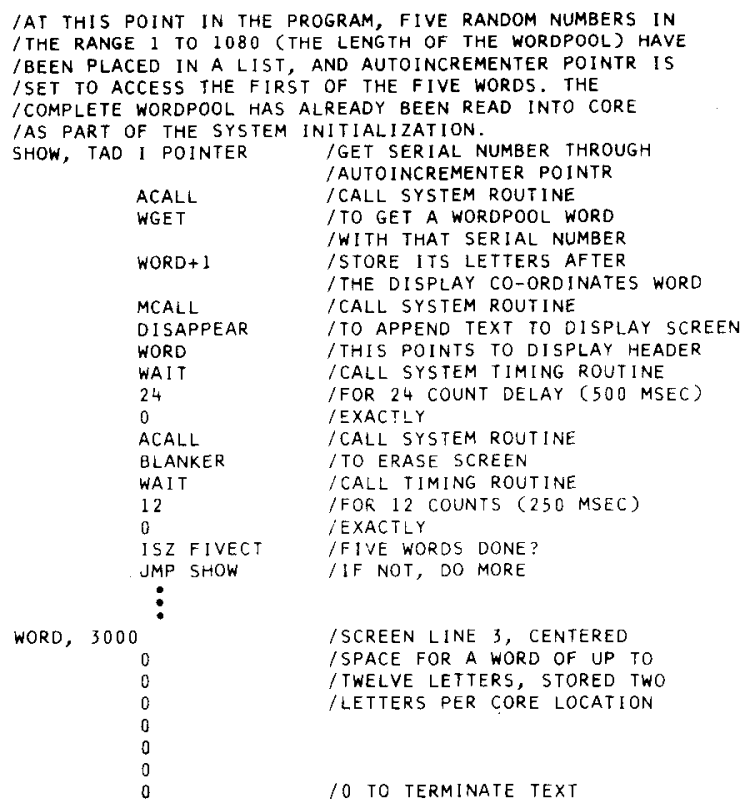

10 TO TERMINATE TEXT

corporate a number of features designed to reduce the number of iterations of the edit, compile, debug cycle. These features fall into three major classes of debugging technique: program intervention, automatic error detection, and program simulation.

Program intervention includes all relatives of that classic debugging tool, ODT. Anyone who has tried to debug an assembler program without ODT, DDT, or a similar tool knows how frustrating and time consuming it can be. We PDP-12 users find ourselves in an intermediate state: Classic PDP-8 ODT will work in a PDP-12, but cannot access upper memory fields, nor can it breakpoint those LINC-mode instructions present on a PDP. 12 but not on a PDP-8. So one of the first tasks in creating FRIVLOS was to rewrite ODT for the expanded PDP-12. The result was KNUCKLE, Kaplan's NUmeric Controller and Keyboard Lab Executive. Extensions to ODT include access to all $32 \mathrm{~K}$ of memory, decimal and negative input and output in addition to the standard positive octal, breakpoints on both PDP and LINC mode instructions, and a major change in the way of handling breakpoints. Classic ODT installs a breakpoint by replacing the contents of the breakpoint location with an ordinary jump back into ODT. KNUCKLE replaces the original contents with a subroutine jump back in to ODT, leaving a record of the actual, as opposed to the intended, breakpoint location, and thus implementing a primitive multiple breakpoint facility. Whether this entry was a scheduled breakpoint, installed by KNUCKLE using an explicit breakpoint command, or an unscheduled one manually installed by the user as if it were a program patch, the full register-saving and -restoring logic is available to the user in returning to his program. Upon encountering a breakpoint, KNUCKLE always prints the actual address at which a break occurred, adding a question mark to indicate an unscheduled breakpoint. This means that it is possible, though not always convenient, to manually overwrite multiple core locations with the subroutine jumps in to KNUCKLE, to see which of several possible program branches is actually taken. These subroutine jump instructions can also be used as primitive error traps to note unexpected branches to program locations that the program logic should be avoiding.

One of the true luxuries of $32 \mathrm{~K}$ of core is that it saves one from certain dangerous temptations. In a $4 \mathrm{~K}$ system, one is often tempted to use ODT during operating system development and then sacrifice it for data space or user programs while running. No such temptation exists in FRIVLOS. KNUCKLE is a permanent part of the system concept, residing in a part of core that cannot be used for user program or data under operating system constraints. We would no more try to program without it than we would try to run FOCAL after deleting all the error messages.

With KNUCKLE a permanent part of FRIVLOS, the chief use of an unscheduled breakpoint is the one in the interrupt handling logic. Every $5 \mathrm{msec}$ the system is interrupted by the clock, software timers are updated, and various $I / O$ devices are serviced as needed. One type of service is seeing if the control-K key has been pressed in the last $5 \mathrm{msec}$. If so, FRIVLOS literally stops the clock and initiates an unscheduled breakpoint into KNUCKLE, allowing the user to make program changes, inspect progress, investigate seemingly infinite loops, or just halt operations while he attends to other business. Unlike the user of FOCAL, a system in which pressing control-C destroys the pushdown list and inhibits the possibility of simple program resumption, the FRIVLOS user need only press " $\mathrm{C}$ " for "continue" to resume program execution at the point where it was stopped. This unscheduled breakpoint can even be used to install or remove other breakpoints, thus allowing real-time decisions about program interruption based on screen displays, typed messages, or other real-time events. In other words, unscheduled entries into KNUCKLE are given the highest interrupt priority in the system and, therefore, constitute a powerful method of program control.

Breakpoints are fine if one knows what parts of the program are worthy of inspection, but may be rather difficult to use in other circumstances. No programmer really wants to breakpoint every four or five instructions throughout a long program, just to watch for possible errors. It is generally more efficient if the system can trap wide classes of errors and report them back to the programmer, so he can work on the program segments leading to the errors. Clearly, one of the best tools for doing this is a small-s-structured source language to detect errors before execution. That being impossible in an assembler-based system, we have adopted the 
next best strategy, automatic error detection: We trap, in software, a wide range of illegal operating system requests and terminate execution with analytical error messages. Conditions trapped include allowing insufficient clock time to complete tasks, calls to nonexistent subroutines, deletion from the display screen of text not on the screen, accesses to nonexistent core, subscripts out of range, and attempted searches of lists whose ends precede their beginnings. Thus, the availability of the operating system routines for common tasks slyly tempts the user into exposing himself to possible error detection. Whenever such an error is encountered, FRIVLOS executes a subroutine jump to its errorhandling routine, which prints both the operating system address where the error was detected (which indicates the type of error) and the user program address at the time the error was detected (the approximate, if not exact, cause of the error). This is like the error message produced by FOCAL, except that the user address is an actual hardware address, not a symbolic statement number. After printing this information, the system branches to KNUCKLE, on the assumption that the first order of business will be to inspect the contents of core for information about the cause of the error.

One major weakness of FRIVLOS is the lack of good facilities for recovery from such system-detected errors. One reason for this difficulty is that only rare errors are so simple as bad constants appearing in an argument list. A more common situation is a bad value being generated by five or six locations worth of bad code, or an incorrect jump being taken a dozen or so locations back. So one difficulty would be unwinding several instructions worth of computation to resume the program at the last known point where nothing had gone wrong. A further difficulty has to do with the structure of the errorhandling routine. Since errors can occur from violation of system timing constraints, and since all $\mathrm{I} / \mathrm{O}$ occurs under control of the system clock, the error handling routine must destroy some of that timing and trace back information in order to print the error messages. Thus it is almost always necessary to rerun a program from the beginning when an error has been detected. Since the error may have occurred 10 or 15 min into a real-time control program, provision has been made in FRIVLOS for speeding up program execution until the errorcausing code is reached, and then running at normal or slow speeds while the correction of the error is tested. This is an example of what we can call debugging by program simulation.

In the sense in which I would like to use the term today, simulation consists of any methods for altering, modifying, or bypassing some program features in order to study other features of more immediate interest. This is not far from the normal use of simulation; we simply run a more useful model of the program than the program itself would be. To facilitate such modeling, the operating system actually exists in two versions, the usual SYSTEM and DSYSTEM, or our debugging system. When we run experiments, for example, we usually assign a separate program-and-data tape to each subject, and invoke a number of protection features to insure that subjects run the various conditions in the correct order and do not overwrite their own or anyone else's data. The construction of such tapes, while necessary for experiments, is a time-consuming nuisance for debugging runs. So, under DSYSTEM, most tape operations are bypassed or simulated; for example, data are written into core buffers for possible KNUCKLE inspection, but these buffers are never transferred onto tape.

To understand the simulation of system timing, it is necessary to know a bit about the FRIVLOS timing structure. The language is of the type recommended by Creelman (1974), in which system events occur during prerequested blocks of time, rather than time being consumed between system events. Three types of WAIT requests exist in FRIVLOS. An ordinary WAIT requests an exact amount of time for completing tasks. A WAIT AT LEAST request guarantees a minimum duration of some event (usually a screen display), but extends the event without an error message until the user completes his response. Another method for terminating time intervals when their tasks are done is to use a WAIT AT MOST command; this command will generate an error if the tasks are not completed in the allowed time, but if they are done sooner, the system proceeds to the next timing interval. Using KNUCKLE, a programmer need change only one core location to automatically convert all wait requests to WAIT AT MOST requests. This has the effect of speeding up his program greatly, while completing at least one screen refresh of every text display in the program. (The effect of seeing 20 separate screen displays in $1 \mathrm{sec}$ can be, to say the least, overwhelming.) To return to normal timing, the user can either install a breakpoint at a critical program segment or interrupt it using the control-K command, and then use KNUCKLE to return all WAIT requests to their ordinary meanings by repairing that one core location.

The chief method of debugging has always been to look at the input and output of a program and see if it appears to function as intended. When the primary output device is a display scope, the programmer may find himself furiously scribbling down the words of a memory task in order to verify later that they fit the required paradigm. Using DSYSTEM, this scribbling is not necessary. By simply setting one of the front panel sense switches, the user can direct that all scope displays also be printed on the Teletype, and this hard copy trace can be enabled and disabled at will. Thus, by stimulating scope display with typewriter copy, we can run a modified program that is more suitable for debugging than was the original.

The ideas I have presented are of varying applicability 
to other systems. The idea that a debugger should be core resident (or quickly swapped in from disk), and should allow easy transfer of program control from itself to the program and back again, is of general applicability. On the other hand, the idea of tracing scope displays on the Teletype is useless for an auditory-event language like PSYCLE or PEPL, but there, perhaps, an option to print on the Teletype all frequencies generated would be an appropriate analogy. If there is any coherent strategy to these debugging aids, it is this: to try to anticipate some of those features that a user might add to his program by dropping in tracing or error-trap statements, were it easy to add such statements, and then to make those features permanent options of the operating system. Since it is not trivial to add statements to the source program, the operating system must provide the opportunities to read between the source statement lines and take action when necessary. If it is worth writing a special system or language to cope with the problems peculiar to one laboratory, it is equally worth writing a special debugging system to cope with the problems peculıar to that special system or language.

\section{REFERENCES}

Creelman, C. D. Softuare management of timing in computer-controlled on-line experiments. Behavior Research Methods \& Instrumentation, 1974, 6, 488-492.

GETTY, D. J The PEPI system for control of experiments by a PDP-8 computer. Behavior Research Methods \& Instrumentation. 1975, 7, 131-136. 